\title{
Thermally assisted and magnetic field driven isostructural distortion of spinel structure and occurrence of polar order in $\mathrm{CoCr}_{2} \mathrm{~S}_{4}$
}

\author{
K. Dey, ${ }^{1}$ A. Karmakar, ${ }^{2}$ A. Indra, ${ }^{1}$ S. Majumdar, ${ }^{1}$ U. Rütt, ${ }^{3}$ O. Gutowski, ${ }^{3}$ M. v. Zimmermann, ${ }^{3}$ and S. Giri ${ }^{1, *}$ \\ ${ }^{1}$ Department of Solid State Physics, Indian Association for the Cultivation of Science, Jadavpur, Kolkata 700032, India \\ ${ }^{2}$ Department of Science and Humanities, Siliguri Government Polytechnic, Siliguri, W.B. 734006, India \\ ${ }^{3}$ Deutsches Elektronen-Synchrotron DESY, Notkestr. 85, 22603 Hamburg, Germany
}

(Received 10 April 2015; revised manuscript received 4 June 2015; published 1 July 2015)

\begin{abstract}
We report appearance of polar order with a ordering temperature $\left(T_{C}\right)$ at $28 \mathrm{~K}$, which is well below the ferrimagnetic order at $225 \mathrm{~K}\left(T_{N}\right)$ for $\mathrm{CoCr}_{2} \mathrm{~S}_{4}$. Intriguingly, the value of spontaneous electric polarization $(P)$ is $\sim 122 \mu \mathrm{C} / \mathrm{m}^{2}$ at $15 \mathrm{~K}$, which is the second largest value in Cr octahedra-based spinels after $\mathrm{CdCr}_{2} \mathrm{~S}_{4}$. Incidentally, the $P$ value is $\sim 60$ times larger than the value of $P$ for the oxide counterpart $\mathrm{CoCr}_{2} \mathrm{O}_{4}$. The significant magnetoelectric coupling is verified from the magnetodielectric response and magnetic field dependent enhancement of $P$. We note that the field-dependent dielectric permittivity scales linearly to the squared magnetization in the low field regime below $\sim 10 \mathrm{kOe}$ as described by the Ginzburg-Landau theory. Synchrotron diffraction studies over a wide temperature range, 15-300 K, illustrate strong magnetoelastic coupling at $T_{N}$ and isostructural distortion at $T_{C}$. Analyses of the diffraction patterns reveal that the occurrence of polar order involves expansion of Co tetrahedra and contraction of Cr octahedra of the spinel structure and these distortions are further enhanced driven by the magnetic field. The delicate interplay between magnetoelastic, magnetoelectric, and electroelastic couplings in $\mathrm{CoCr}_{2} \mathrm{~S}_{4}$ proposes the system as a potential candidate in multiferroics.
\end{abstract}

DOI: 10.1103/PhysRevB.92.024401

PACS number(s): 75.80.+q, 77.80.-e

\section{INTRODUCTION}

Multiferroics are the rare class of chemically single-phase materials where more than two ferroic order parameters coexist concomitantly. These materials are highly promising for technological applications because of intriguing coupling phenomena between the ferroic orders. The significant magnetoelectric coupling in a chemically single-phase material is extremely promising, because one can control magnetization by applying electric field or electric polarization by the magnetic field [1-3]. Unfortunately, evidence of ferroelectric order is very rare compared to the magnetic orders, which have been explored extensively in the history of science. Out of ferroelectricity, magnetic order driven ferroelectricity, termed as improper ferroelectricity, attracts interest for the intriguing magnetoelectric coupling [4]. From the technological perspective, enhancement of magnetic and ferroelectric polarizations with a strong magnetoelectric coupling as well as increase of multiferroic ordering temperatures close to room temperature are the primary goal in this field.

Recent discoveries of multiferroic order in the $A \mathrm{Cr}_{2} \mathrm{O}_{4}$ ( $A=\mathrm{Mn}, \mathrm{Fe}, \mathrm{Co}, \mathrm{Ni}$ ) family with spinel structure has attracted significant attention [5-11]. The first report of improper ferroelectricity in this series was reported in $\mathrm{CoCr}_{2} \mathrm{O}_{4}$ [7]. Interestingly, magnetic field driven polarization reversal was reported in this compound [8]. About 10 times enhancement of ferroelectric polarization $(P)$ was obtained in $\mathrm{FeCr}_{2} \mathrm{O}_{4}$ compared to the value for $\mathrm{CoCr}_{2} \mathrm{O}_{4}$ where additional distortion of spinel structure ascribed to the Jahn-Teller active $\mathrm{Fe}^{2+}$ ion was proposed for this significant increase [6]. A dynamical structural disorder-driven relaxor-type ferroelectricity was proposed in $\mathrm{Co}_{1-x} \mathrm{Ni}_{x} \mathrm{Cr}_{2} \mathrm{O}_{4}$ solid solutions with $x=0.2$, 0.4 , and 0.6 [11]. Comparable to the value of $P$ observed in

*sspsg2@iacs.res.in
$\mathrm{FeCr}_{2} \mathrm{O}_{4}$, a large value of $P$ was also reported for $\mathrm{NiCr}_{2} \mathrm{O}_{4}$ and, intriguingly, the appearance of ferroelectricity has been proposed in collinear magnetic structure [10]. Recently, ferroelectricity was reported in $\mathrm{MnCr}_{2} \mathrm{O}_{4}$ [5]. The release of topological frustration occurred due to the pyrochlore structure of Cr-sublattice, short-range conical magnetic order, and the emergence of ferroelectricity have been correlated to the structural instability as observed from meticulous synchrotron diffraction studies. Thus the origin of ferroelectricity in $A \mathrm{Cr}_{2} \mathrm{O}_{4}$ is still a debatable issue.

Compared to the oxide spinels, sulphospinels are rather less investigated, specially, in the context of multiferroics. The spinel $\mathrm{CdCr}_{2} \mathrm{~S}_{4}$ is the first unique compound that exhibited ferroelectricity and ferromagnetism concomitantly [12]. It has been suggested that geometrical frustration in the pyrochlore structure formed by the $\mathrm{Cr}$ sublattice plays a vital role for the relaxor ferroelectricity in $\mathrm{CdCr}_{2} \mathrm{~S}_{4}[12,13]$. Coexistence of colossal magnetocapacitance and magnetoresistance associated with the ferroelectricity, as observed from the hysteretic polarization $(P-E)$ loop, has been reported in $\mathrm{HgCr}_{2} \mathrm{~S}_{4}$, although ferroelectricity could not be detected from the pyroelectric current measurements [14]. Recently, reasonably large ferroelectricity was reported in $\mathrm{FeCr}_{2} \mathrm{~S}_{4}$ [15]. Interestingly, the ferroelectricity had two components, which were attributed to the noncollinear conical spin order and Jahn-Teller distortion as induced by the lattice symmetry breaking. Apart from these rare consequences of multiferroicity in sulphospinels, spin-driven ferroelectricity has also been reported in very few chromium disulphides, $\mathrm{AgCrS}_{2}[16,17]$ and $\mathrm{CuCrS}_{2}$ [18]. The helimagnetic order has been suggested for the occurrence of ferroelectricity in $\mathrm{AgCrS}_{2}$ and $\mathrm{CuCrS}_{2}$. An interesting scenario of memory effect in dielectricity and magnetic field induced rejuvenation were reported for $\mathrm{CuCrS}_{2}$ above the ferroelectric transition temperature [18]. The geometrical frustration in the antiferromagnetically coupled two-dimensional (2D) triangular lattice was proposed for the dielectric memory effect. 
The sulphospinel compound $\mathrm{CoCr}_{2} \mathrm{~S}_{4}$ (CCS) of our interest has been less explored so far. A bulk magnetization study exhibited ferromagneticlike transition at $\sim 225 \mathrm{~K}$ [19-21]. A strong hybridization between $\mathrm{Co}^{2+}$ and $\mathrm{Cr}^{3+}$ has been proposed for a high value of $T_{N}$ in CCS [22]. The magnetooptical properties have been investigated extensively in CCS [22-28]. The possible collinear or noncollinear spin dynamics has been discussed between $A$-site and $B$-site magnetic ions in an $A B_{2} \mathrm{~S}_{4}$-type spinel structure by considering the contribution of relative magnitude of magnetocrystalline anisotropy at the $A$ or $B$ site to the exchange interactions [27,28].

In this article we report another rare occurrence of spontaneous electric polarization in CCS. Intriguingly, the value of electric polarization $(P)$ has a $\sim 60$ times magnified value compared to that observed in the oxide counterpart $\mathrm{CoCr}_{2} \mathrm{O}_{4}$ (CCO) [7]. The value of $P$ is appreciably larger than the enhanced $P$ value due to $A$-site [11] as well as $B$-site [29] doping in CCO. Incidentally, the value of electric polarization with $P \sim 122 \mu \mathrm{C} / \mathrm{m}^{2}$ at $15 \mathrm{~K}$ for $4 \mathrm{kV} / \mathrm{cm}$ poling field is the largest in ferroelectric spinels [14-18], except for $\mathrm{CdCr}_{2} \mathrm{~S}_{4}$ [12]. The magnetic field dependent $P$ reveals significant magnetoelectric coupling while the magnetodielectric response scales linearly to the squared magnetization below $\sim 10 \mathrm{kOe}$ as described by the Ginzburg-Landau theory. X-ray synchrotron diffraction studies clearly reveal strong isostructural magnetoelastic coupling at the magnetic ordering temperature $\left(T_{N}\right)$. With decreasing temperature the significant distortions of $\mathrm{CoS}_{4}$ tetrahedra and $\mathrm{CrS}_{6}$ octahedra are observed at the ferroelectric ordering temperature $\left(T_{C}\right)$. These distortions are further magnified upon application of magnetic field, which is evident from the unique experiment of synchrotron diffraction studies in magnetic field. We suggest that magnetic field induced enhancement of $P$ is correlated to the expansion of $\mathrm{CoS}_{4}$ tetrahedra in the spinel structure. The coupling between spin, charge, and lattice degrees of freedom is discussed for realizing unexplored improper multiferroic order in CCS.

\section{EXPERIMENTAL DETAILS}

The Co (99.9\%, Aldrich), Cr (99.5\%, Aldrich), and S (99.98\%, Aldrich) powders are used as starting materials for preparing a polycrystalline specimen, $\mathrm{CoCr}_{2} \mathrm{~S}_{4}$ using solidstate reaction [21]. All the heat treatments are done in an evacuated $\left(<10^{-4}\right.$ torr) quartz tube and final heat treatment is done at $1173 \mathrm{~K}$ with excess S. Single-phase chemical composition is confirmed by the X-ray diffraction studies at room temperature recorded in a SEIFERT $x$-ray diffractometer (Model: XRAY3000P) using $\mathrm{Cu} \mathrm{K} \alpha$ radiation, which is further confirmed by the high-energy synchrotron radiation studies at beam line P07 of PETRA III in Hamburg, Germany. The used wavelength is $0.1252 \AA(99 \mathrm{keV})$. The synchrotron powder diffraction data are analyzed using Rietveld refinement with a commercially available software MAUD (materials analysis using diffraction), which is further checked by the FULLPROF software. A $100 \mathrm{kOe}$ horizontal field magnet (Cryogenics, UK) has been employed for high-energy $\mathrm{x}$-ray diffraction studies under magnetic field.

A powder sample pressed into a pellet is used for the resistivity measurement using a source meter (Keithley, model 2400) and nanovoltmeter (Keithley, model 2182A) for low resistance. Dielectric permittivity is recorded in a E4980A LCR meter (Agilent Technologies, USA) equipped with a cryogen-free cryocooler (JANIS, USA) on the compressed powder specimens in the range 7-300 K. The pyroelectric current $\left(I_{p}\right)$ is recorded in an electrometer (Keithley, model 6517B) at a constant temperature sweep rate. The $I_{p}$ is integrated with time for obtaining $P$. The poling electric fields are applied during cooling processes and all the measurements are carried out in the warming mode. For measurement of $P$ in field, magnetic field is applied during poling process and $I_{p}$ is measured in the absence of magnetic and electric fields. Before measurement of $I_{p}$ electrical connections are short circuited and rested for sufficiently long time. Electrical contacts are fabricated using an air drying silver paint. The ac susceptibility and dc magnetization are measured in a commercial magnetometer of Quantum Design (MPMS, evercool).

\section{EXPERIMENTAL RESULTS AND DISCUSSIONS}

Thermal variation resistivity $(\rho)$ in a semilog plot is depicted in Fig. 1(a). With decreasing temperature the value of $\rho$ increases and stabilizes below $\sim 150 \mathrm{~K}$, which is followed by another small increase in $\rho$ below $\sim 60 \mathrm{~K}$ as evident in the inset of the figure. The value of $\rho$ at $10 \mathrm{~K}$ is $\sim 0.03 \mathrm{G} \Omega-\mathrm{cm}$, which is close to that reported for sulphur stoichiometric compound [30]. Thermal variation of the real part of ac susceptibility $\left(\chi^{\prime}\right)$ is depicted in Fig. 1(b). It shows a sharp rise pointing to a ferrimagnetic transition at $T_{N}=225 \mathrm{~K}$ in accordance with the previous reports [19-21]. With decreasing temperature below $T_{N}$ a broadened shoulder appears around $60 \mathrm{~K}$. This is further indicated by the $T$ derivative of $\chi^{\prime}$, $d \chi^{\prime} / d T$ as shown in the inset of the figure. Temperature variation of the zero-field-cooled (ZFC) and field-cooled (FC) magnetization $(M)$ recorded at $H=100 \mathrm{Oe}$ is displayed in Fig. 1(c). The ZFC magnetization depicts quite a sharp increase at $T_{N}$. The ZFC magnetization also exhibits a diffused shoulder around $60 \mathrm{~K}$. We note that the low- $T$ anomaly observed in the ac and dc magnetization is also manifested in the
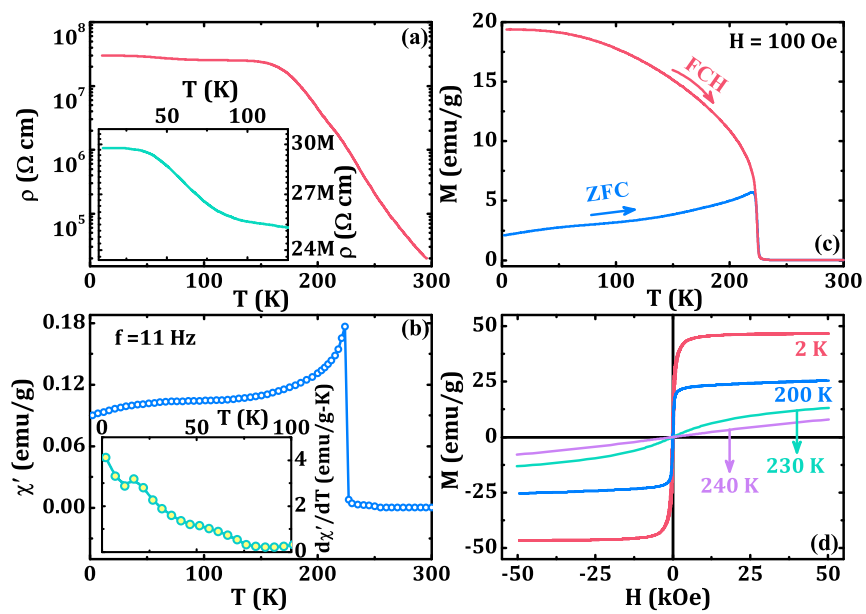

FIG. 1. (Color online) (a) Thermal variation resistivity $(\rho)$. Inset highlights the low- $T$ region. (b) Real part of ac susceptibility $\left(\chi^{\prime}\right)$ with $T$. Inset shows $T$ derivative of $\chi^{\prime}$ with $T$ in the low- $T$ region. (c) FC-ZFC magnetization with $T$. (d) Magnetic hysteresis loops recorded at various $T$. 

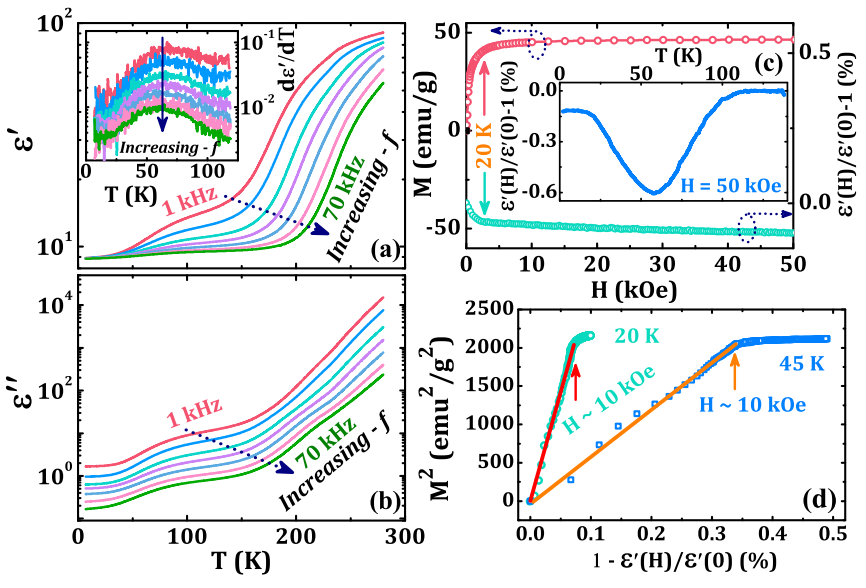

FIG. 2. (Color online) Thermal variations of (a) real $\left(\epsilon^{\prime}\right)$ and (b) imaginary $\left(\epsilon^{\prime \prime}\right)$ parts of dielectric permittivity at different $f$. Inset of (a) shows the low- $T$ region of $d \epsilon^{\prime} / d T$, highlighting the peaks. Arrow indicates no shift of peak position at different frequencies, $f$. (c) Magnetization $(M)$ curve and magnetodielectric response $\left[\epsilon^{\prime}(H) / \epsilon^{\prime}(0)-1\right]$ with $H$. Inset demonstrates thermal variation of $\epsilon^{\prime}(H) / \epsilon^{\prime}(0)-1$. (d) Plot of $M^{2}$ vs $1-\epsilon^{\prime}(H) / \epsilon^{\prime}(0)$ at 20 and $45 \mathrm{~K}$.

thermal variation of $\rho$ as well as the lattice constant (Fig. 5). We further note that the onset of polar order starts around this temperature (Fig. 3) as discussed elsewhere in the text. Magnetic hysteresis loops measured at selective temperatures are shown in Fig. 1(d). The loop at $2 \mathrm{~K}$ displays typical soft ferromagneticlike character with a small coercivity of $\sim 100$ Oe. With increasing $T$ the values of $M$ at $50 \mathrm{kOe}$ decrease and a linear magnetization curve is observed in the paramagnetic region at $240 \mathrm{~K}$.

Dielectric permittivity $(\epsilon)$ is measured at different frequencies $(f)$. The real $\left(\epsilon^{\prime}\right)$ and imaginary $\left(\epsilon^{\prime \prime}\right)$ components of $\epsilon$ with $T$ are depicted in Figs. 2(a) and 2(b), respectively. The $\epsilon^{\prime}(T)$ displays a steplike decrease with decreasing temperature, which is followed by another steplike decrease below $100 \mathrm{~K}$. The $d \epsilon^{\prime} / d T$ with $T$ in the low- $T$ region is depicted in the inset of Fig. 2(a), which exhibits a peak around $\sim 60 \mathrm{~K}$. Significantly, the peak position does not change convincingly at different $f$. This confirms that the emergence of the peak in $d \epsilon^{\prime} / d T$ does not involve grain boundary effect. We observe magnetodielectric (MD) response defined as, $\epsilon^{\prime}(H) / \epsilon^{\prime}(0)-1$, although we could not detect magnetoresistance below $\sim 120 \mathrm{~K}$ beyond the instrumental limitations $(\sim 0.05 \%$ of the value of resistance). In accordance with the reported large magnetoresistance, we also observe significant magnetoresistance up to $\sim 6.8 \%$ at $T_{N}$ and it nearly vanishes below $\sim 150 \mathrm{~K}$ [30]. The existence of MD without convincing contribution from the magnetoresistance is vital, which indicates the intrinsic magnetoelectric coupling. Thermal variation of $\operatorname{MD}(\%)$ is shown in the inset of Fig. 2(c), which exhibits a maximum around $60 \mathrm{~K}$. The maximum value around $60 \mathrm{~K}$, at which a peak in $d \epsilon^{\prime} / d T$ is also observed, is considerable $(0.6 \%$ in $50 \mathrm{kOe}$ ) and comparable to that observed for $\mathrm{BiMnO}_{3}$ [31]. The value is rather significantly larger than the value for other spinel oxides $M \mathrm{Cr}_{2} \mathrm{O}_{4}(M=\mathrm{Mn}, \mathrm{Co}$, and $\mathrm{Ni})[32,33]$. The change in the sample dimension or lattice constant driven by the magnetic field, i.e., magnetostriction, may lead to the observed magnetocapacitance effect. To confirm it, the magnetic field dependent lattice constant, as obtained from the Rietveld refinement of the synchrotron diffraction data recorded in magnetic field at a particular temperature, is estimated to be $\Delta a / a \sim 1.7 \times 10^{-5}$ at $45 \mathrm{~K}$ and $50 \mathrm{kOe}$. Thus the estimated change in $\epsilon$ ascribed to the magnetostriction is $\sim 1.7 \times 10^{-3} \%$, which is two orders of magnitude smaller than the observed value of $\mathrm{MD}(\%) \sim 0.5 \%$ at $45 \mathrm{~K}$ and $50 \mathrm{kOe}$. This points to the fact that magnetostriction does not contribute significantly to the MD value. Magnetic field dependent MD and magnetization are also depicted, for example at $20 \mathrm{~K}$, in Fig. 2(c). The MD or magnetocapacitance effect can be phenomenologically described by a simple Ginzburg-Landau theory for the second-order phase transition and is attributed to the ME coupling term $\gamma P^{2} M^{2}$ in the thermodynamic potential $(\Phi)$ given by

$$
\begin{aligned}
\Phi= & \Phi_{0}+\alpha P^{2}+\frac{\beta}{2} P^{4}-P E+\alpha^{\prime} M^{2}+\frac{\beta^{\prime}}{2} M^{4} \\
& -M H+\gamma P^{2} M^{2},
\end{aligned}
$$

where $\alpha, \beta, \alpha^{\prime}, \beta^{\prime}$, and $\gamma$ are the constants and functions of temperature. The influence of magnetic order on the magnetic field driven magnetocapacitance can be followed by the linear MD vs $M^{2}$ curve in the low field region [31-33]. Here, the linear $-\left[\epsilon^{\prime}(H) / \epsilon^{\prime}(0)-1\right](\%)$ vs $M^{2}$ plots at 20 and $45 \mathrm{~K}$ are depicted in Fig. 2(d). We note that at both temperatures the linearity of the curve holds below $\sim 10 \mathrm{kOe}$. This illustrates that the ME coupling term $\gamma P^{2} M^{2}$ of the Ginzburg-Landau theory [Eq. (1)] is significant for CCS as reported in $M \mathrm{Cr}_{2} \mathrm{O}_{4}$ $(M=\mathrm{Mn}, \mathrm{Co}, \mathrm{Ni})[32,33]$.

To confirm the origin of ME coupling correlated to the occurrence of polar order, $I_{p}$ is recorded with $T$ at different conditions. A peak in $I_{p}(T)$ is observed as evident in Fig. 3(a) recorded at different heating rates for $2 \mathrm{kV} / \mathrm{cm}$ poling field. Time-integrated $I_{p}$ provides $P$ as a function of $T$, which is depicted in Fig. 3(b) for $\pm 2 \mathrm{kV} / \mathrm{cm}$ poling field. Reversal
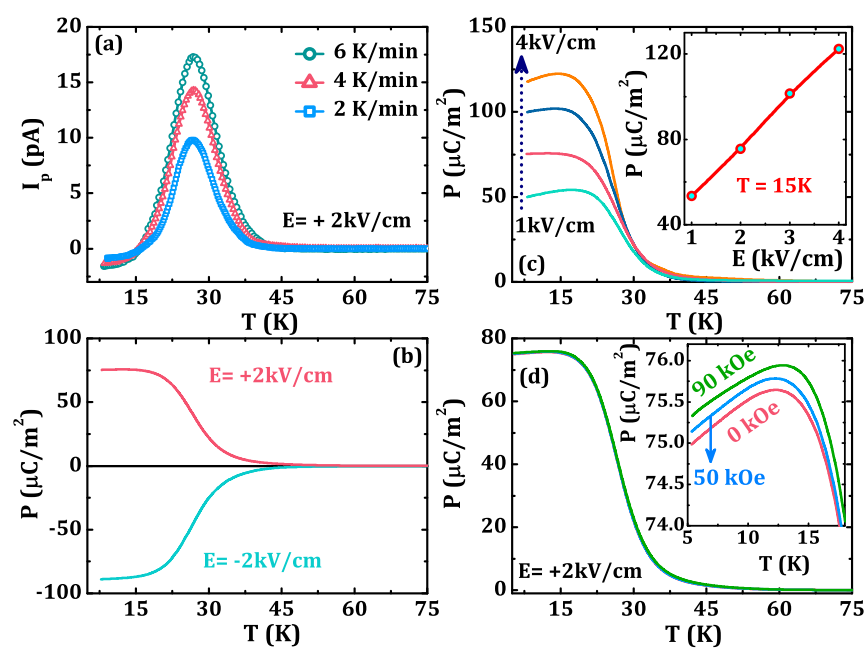

FIG. 3. (Color online) (a) $T$ variation of pyroelectric current $\left(I_{p}\right)$ at different thermal sweep rates for $2 \mathrm{kV} / \mathrm{cm}$ poling field. Thermal variations of (b) electric polarization $(P)$ for $\pm 2 \mathrm{kV} / \mathrm{cm}$ poling field, (c) $P$ for different poling fields. Inset shows the plot of $P$ with poling field $(E)$, and (d) $P$ in $H=0,50$, and $90 \mathrm{kOe}$. Inset highlights the low- $T$ data. 
of $P$ due to a change in sign of poling field confirms ferroelectric order and suggests that CCS is a new improper multiferroic compound. We note that ferroelectric transition $\left(T_{C}\right)$ occurs at $\sim 28 \mathrm{~K}$, around which any peak in $\epsilon(T)$ is not apparent. However, the results are consistent with that observed for few improper ferroelectrics [34-39] including spinel $\mathrm{NiCr}_{2} \mathrm{O}_{4}$ [10] and $\mathrm{LuFe}_{2} \mathrm{O}_{4}[40,41]$. Here, a peak in $d \epsilon^{\prime} / d T$ is observed, around which polarization occurs and a maximum in MD response is also observed, which is analogous to that reported in ferroelectric $\mathrm{Dy}_{2} \mathrm{BaNiO}_{5}$ [38]. Poling-fielddependent polarization results are depicted in Fig. 3(c). The inset shows poling-field $(E)$-dependent $P$ at $15 \mathrm{~K}$, illustrating that $P$ does not saturate even at $4 \mathrm{kV} / \mathrm{cm}$ field. Importantly, the value of $P$ is $\sim 60$ times larger than that of the value for the oxide counterpart CCO [7]. Moreover, the magnitude of $P$ provides the second-highest value in the reported $\mathrm{Cr}$ spinels [14-18]. The polarization measured in $H$ is shown in Fig. 3(d). The $P(T)$ results below $T_{C}$ are further highlighted in the inset of the figure. The results illustrate that $P$ increases with increasing $H$ up to $\sim 0.4 \%$ at $7 \mathrm{~K}$ for $H=90 \mathrm{kOe}$ and confirms significant magnetoelectric coupling, in accordance with that observed in the MD response.

The structural properties are thoroughly investigated by synchrotron $\mathrm{x}$-ray diffraction studies in the temperature range, $15-300 \mathrm{~K}$. The powder diffraction pattern recorded at $300 \mathrm{~K}$ is depicted in Fig. 4(a). The diffraction pattern is analyzed using Rietveld refinement by taking into account of $F d \overline{3} m$ space group with atomic positions, Co $\left(\begin{array}{lll}0 & 0 & 0\end{array}\right), \mathrm{Cr}$ $(0.625,0.625,0.625)$, and $\mathrm{S}(x=y=z)$, which is consistent with that observed for most of the spinel oxides including CCO and chalcogenides [22,42]. The fit using Rietveld refinement of
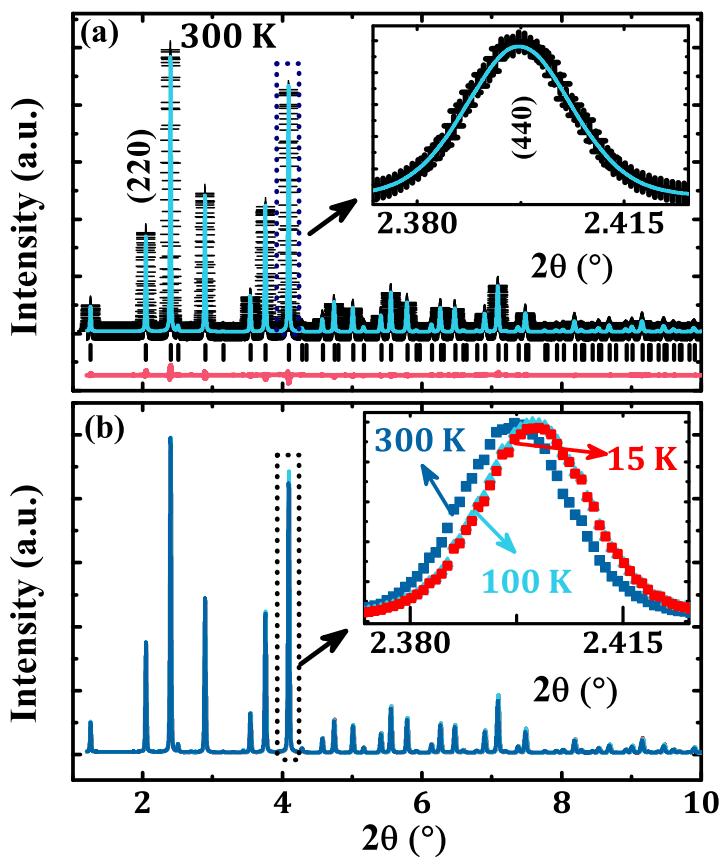

FIG. 4. (Color online) Rietveld refinement of x-ray powder diffraction pattern (black symbols) at 300 (a). Solid curve demonstrates the fit. Inset exhibits the fit of (440) diffraction peak. (b) X-ray diffraction patterns at 300, 100, and $15 \mathrm{~K}$. Inset depicts (440) diffraction peak at three temperatures. the diffraction pattern (symbol) is illustrated by the continuous curve in Fig. 4(a). The inset of the figure clearly demonstrates the fit of the (440) diffraction peak by the continuous curve. The bars below the diffraction pattern represent the diffraction peak positions and the difference plot is shown at the bottom of Fig. 4(a). The reliability parameters, $R_{w}(\sim 3.31), R_{\exp }(\sim 1.32)$, and $\sigma(\sim 2.90)$ at $300 \mathrm{~K}$ are reasonably small.

Figure 4(b) depicts the diffraction patterns at 15, 100, and $300 \mathrm{~K}$. These are the three characteristic temperatures at paramagnetic, below ferrimagnetic and ferroelectric transition temperatures, respectively. The inset of Fig. 4(b) highlights the (440) diffraction peaks at three temperatures. The results do not show any change of shape of the peak, except for the shift of the peak position toward high $2 \theta$ value as expected due to lattice contraction with decreasing temperature. This indicates absence of symmetry lowering at the characteristic temperatures as also observed for CCO $[43,44]$. Therefore, all the diffraction patterns over $15-300 \mathrm{~K}$ temperature range are refined using the $F d \overline{3} m$ space group. The integrated intensity of a single (220) diffraction peak is plotted with $T$ as shown in Fig. 5(a). A considerable change of intensity is observed at $T_{N}$ and ferroelectric $T_{C}$. We note that the intensity change is nearly doubled at $T_{C}$ compared to that observed at $T_{N}$. Intriguingly, the intensity change at $T_{C}$ is $\sim 1.4 \%$, which is much larger than the results observed for improper ferroelectric $\mathrm{MnCr}_{2} \mathrm{O}_{4}$ [5], $\mathrm{Li}_{0.99} \mathrm{Cu}_{0.01} \mathrm{CrO}_{2}$ [45], and $\mathrm{LiCr}_{0.99} \mathrm{Fe}_{0.01} \mathrm{O}_{2}$ [46]. Positions
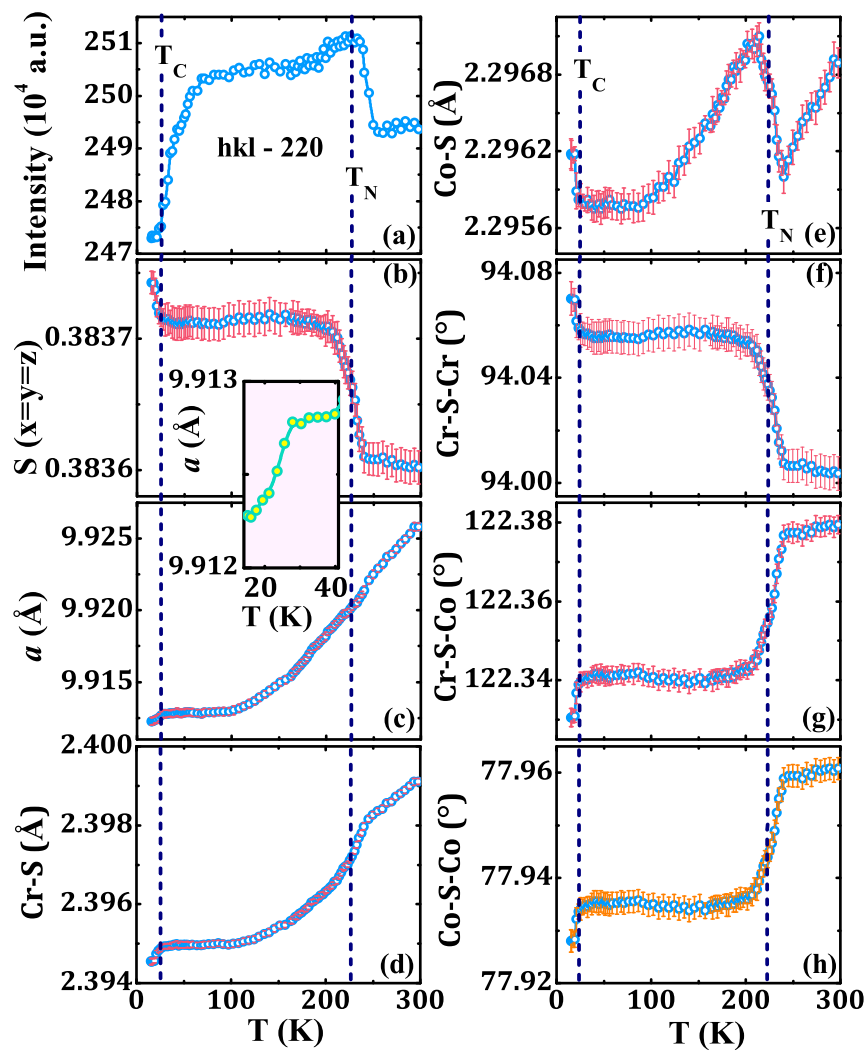

FIG. 5. (Color online) Temperature variations of the (a) integrated intensity of (220) peak, (b) S displacement, and (c) lattice constant (a). Inset of (b) and (c) shows the low- $T$ region of $a . T$ variation of (d) Cr-S bond length, (e) Co-S bond length, (f) Cr-S-Cr, (g) Cr-S-Co, and (h) Co-S-Co bond angles. Vertical lines at 225 and $28 \mathrm{~K}$ demonstrate $T_{N}$ and ferroelectric $T_{C}$, respectively. 
of $T_{N}$ and $T_{C}$ are illustrated by the vertical lines in Fig. 5. These significant changes are also manifested in the refined parameters, such as the coordinate of $\mathrm{S}$ and lattice constant $(a)$ as depicted in Figs. 5(b) and 5(c), respectively. The apparent signature in $a(T)$ around $T_{N}$ points to the significant magnetoelastic coupling. We note that the signature of transition at $T_{C}$ is manifested by a considerable change in $a(T)$ at $T_{C}$, which is much stronger than that observed at $T_{N}$. The $a(T)$ decreases quite sharply below $T_{C}$, which is highlighted in the inset of Figs. 5(b) and 5(c).

In the spinel structure with $F d \overline{3} m$ space group $\mathrm{Co}^{2+}$ and $\mathrm{Cr}^{3+}$ ions reside at the tetrahedral and octahedral positions, respectively, where corners of the Co tetrahedra and $\mathrm{Cr}$ octahedra are occupied by $\mathrm{S}$ atoms. Figures $5(\mathrm{~d})$ and $5(\mathrm{e})$ depict thermal variations of $\mathrm{Cr}-\mathrm{S}\left(d_{\mathrm{Cr}-\mathrm{S}}\right)$ and $\mathrm{Co}-\mathrm{S}\left(d_{\mathrm{Co}-\mathrm{S}}\right)$ bond lengths, respectively. More convincing signatures of $T_{N}$ and $T_{C}$ are evident in the thermal variation of $d_{\mathrm{Cr}-\mathrm{S}}$ and $d_{\mathrm{Co}-\mathrm{S}}$. With decreasing temperature $d_{\mathrm{Cr}-\mathrm{s}}$ decreases almost linearly and this trend becomes faster while approaching toward $T_{N}$. Below $\sim 100 \mathrm{~K}, d_{\mathrm{Cr}-\mathrm{S}}$ becomes temperature independent and it decreases quite sharply below $T_{C}$. Unlike the thermal variation of $d_{\text {Cr-s }}, d_{\text {Co-S }}$ exhibits an anomalous increase $(\sim 0.05 \%)$ around $T_{N}$ in the range, $205-220 \mathrm{~K}$, below which it decreases sharply and becomes nearly temperature independent below $\sim 80 \mathrm{~K}$. Intriguingly, a rapid anomalous increase of $d_{\mathrm{Co}-\mathrm{S}}$ is observed in contrast to the rapid fall of $d_{\mathrm{Cr}-\mathrm{S}}$ below $T_{C}$. The results below $T_{C}$ are significant, because the appearance of ferroelectricity involves expansion of $\mathrm{CoS}_{4}$ tetrahedra and contraction of $\mathrm{CrS}_{6}$ octahedra. The $\mathrm{S}$ displacement also leads to the almost steplike changes in the Cr-S-Cr, Cr-S-Co, and Co-S-Co bond angles at $T_{N}$ and $T_{C}$ as depicted in Figs. 5(f)-5(h), respectively. Temperature variation behaves similarly for $\mathrm{Cr}-\mathrm{S}-\mathrm{Co}$ and Co-S-Co while Cr-S-Cr shows the opposite behavior.

We perform a unique experiment of synchrotron diffraction studies under the magnetic field. The x-ray diffraction data at $45 \mathrm{~K}$ recorded in $H=0$ and $100 \mathrm{kOe}$ are depicted in Fig. 6(a). A change in (440) diffraction peak profile due to application of $100 \mathrm{kOe}$ field is shown in the inset of the figure. The continuous curve demonstrates the satisfactory fit using Rietveld refinement. The values of $d_{\mathrm{Co}-\mathrm{S}}$ and $d_{\mathrm{Cr}-\mathrm{S}}$ with $H$, as obtained from the refinement, are displayed in Fig. 6(b). The $d_{\mathrm{Cr}-\mathrm{S}}$ decreases while $d_{\mathrm{Co}-\mathrm{S}}$ increases upon application of magnetic field. We note that the increase of $d_{\mathrm{Co}-\mathrm{S}}$ is $\sim 0.5 \%$, which is nearly double that of the decrease of $d_{\mathrm{Cr}-\mathrm{S}}(\sim 0.25 \%)$ for $H=100 \mathrm{kOe}$. This is consistent with the distortion of bond lengths with decreasing temperature below ferroelectric $T_{C}$ as evident in Figs. 5(d) and 5(e) and points to the fact that thermally assisted and magnetic field driven distortion of $d_{\mathrm{Co}-\mathrm{S}}$ is much stronger than the effect on $d_{\mathrm{Cr}-\mathrm{S}}$. Direct observation of magnetic field dependent structural distortion in the current observation is significant, which is consistent with the proposed spin-orbit coupling in $\mathrm{CoCr}_{2} \mathrm{~S}_{4}$ [47].

We note that the emergence of ferroelectricity involves significant isostructural distortion of the centrosymmetric $F d \overline{3} m$ structure, as also observed similarly in multiferroic oxide counterpart $\mathrm{CCO}[7,8]$ and $\mathrm{MnCr}_{2} \mathrm{O}_{4}$ [5]. As illustrated in Fig. 7(a) $\mathrm{CoS}_{4}$ tetrahedra are connected through the $\mathrm{CrS}_{6}$ octahedra or vice versa. The rapid $\mathrm{S}$ displacement below ferroelectric $T_{C}$ leads to the expansion of $\mathrm{CoS}_{4}$ tetrahedra and contraction of $\mathrm{CrS}_{6}$ octahedra as shown in Figs. 7(b)
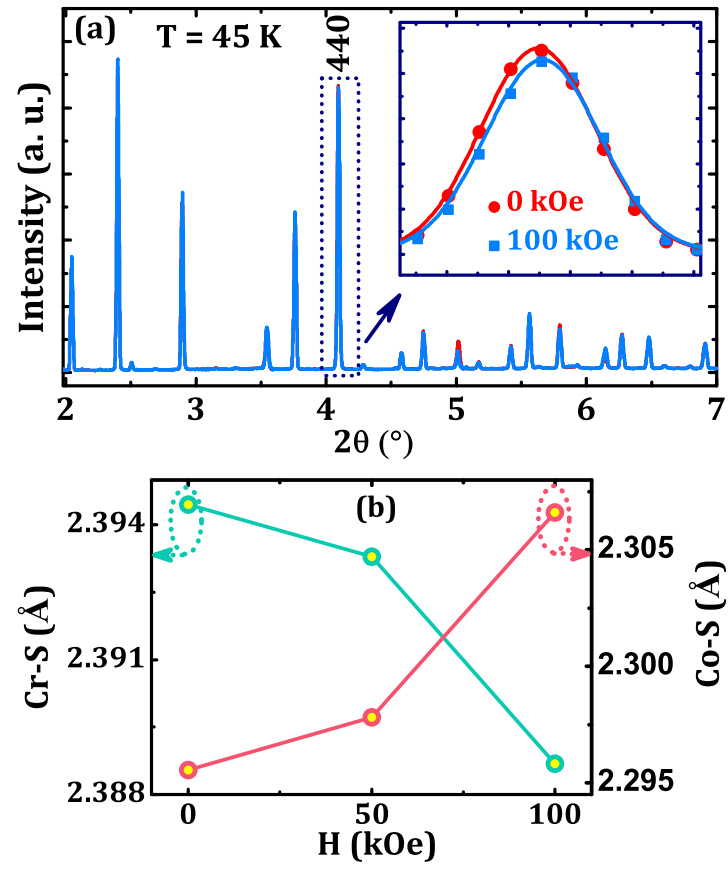

FIG. 6. (Color online) (a) At $45 \mathrm{~K}$ x-ray diffraction patterns recorded at $H=0$ and $100 \mathrm{kOe}$. Inset shows the (440) diffraction peak. (b) $\mathrm{Cr}-\mathrm{S}$ and $\mathrm{Co}-\mathrm{S}$ bond lengths with $H$ as obtained from the refinement.

and 7(c), respectively. Analogous to the thermally assisted behavior, application of the magnetic field further deforms the $\mathrm{CoS}_{4}$ tetrahedra and $\mathrm{CrS}_{6}$ octahedra in a similar manner. We note that the value of $P$ increases upon application of the magnetic field. This indicates that magnetic field driven enhancement of $P$ is somewhat correlated to the increase of isostructural deformation of the spinal structure. Nevertheless, only isostructural distortion of the centrosymmetric structure can not explain the occurrence of ferroelectricity. Thus, the emergence of ferroelectricity must involve a crucial role of magnetic order and CCS is probably an improper ferroelectric material. So far improper ferroelectricities in spinel oxides and sulphides have been proposed either due to spiral spin order as observed in $\mathrm{CCO}$ [7,8], $\mathrm{MnCr}_{2} \mathrm{O}_{4}$ [5], $\mathrm{HgCr}_{2} \mathrm{~S}_{4}$ [14], and $\mathrm{FeCr}_{2} \mathrm{~S}_{4}$ [15], or due to collinear magnetic structure having significant magnetostriction as also reported in $\mathrm{NiCr}_{2} \mathrm{O}_{4}$ and $\mathrm{FeCr}_{2} \mathrm{O}_{4}[6,10]$. In the case of multiferroic $\mathrm{NiCr}_{2} \mathrm{O}_{4}$ and $\mathrm{FeCr}_{2} \mathrm{~S}_{4}$ the magnetostriction engages structural transition to

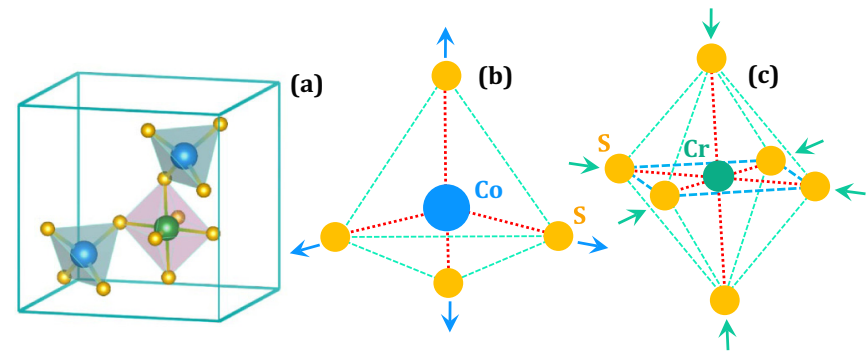

FIG. 7. (Color online) (a) An example of the linkage of $\mathrm{CoS}_{4}$ tetrahedra through a $\mathrm{CrS}_{6}$ octahedron. Schematic representation of (b) expansion of $\mathrm{CoS}_{4}$ tetrahedron and (c) contraction of $\mathrm{CrS}_{6}$ octahedron. 
the lower crystal symmetry [10]. For example, ferroelectricity of $\mathrm{NiCr}_{2} \mathrm{O}_{4}$ involves symmetry lowering to $F d d d$ space group [48]. In the current investigation strong isostructural transition is suggested to be correlated with the appearance of spiral spin order below $T_{C}$ as pointed out in CCO and $\mathrm{MnCr}_{2} \mathrm{O}_{4}$ [49]. The unexplored low-temperature neutron diffraction studies are proposed to settle this issue.

The significantly large magnitude of $P$ in CCS among $\mathrm{Cr}$ octahedra-based spinels is one of the central issues in the current investigation. Importantly, the value of $P$ is $\sim 60$ times enhanced than the value for the oxide counterpart CCO. Intriguingly, the markedly enhanced $P$ of CCS is associated with the $\sim 12$ times enhancement of magnetization value at $2 \mathrm{~K}$ compared to the oxide counterpart CCO along the [111] direction [22]. We note that these results are associated with the significantly different lattice constant as well as covalency between these two. The covalency has been found considerably larger for CCS compared to CCO due to S ligand. The large covalency significantly influenced the optical conductivity spectra of CCS [22]. The magnitude of electrical conductivity has been found to be comparable in magnitude for both the CCS and CCO below ferroelectric $T_{C}$. The only difference of conductivity between these two is the temperature-dependent behavior of $\rho$, where $\rho(T)$ stabilizes below $T_{C}$ for CCS in contrast to slowly increasing trend for $\mathrm{CCO}$ with decreasing temperature. At least partially, this might be correlated to the development of electric polarization, where $\rho(T)$ contributes to the more lossy component to the polar order in $\mathrm{CCO}$ than CCS. In accordance with the major spinel compounds, the lattice constant of CCS at $15 \mathrm{~K}$ [9.9122(8) $\AA$ ] is significantly larger than that of the value for $\mathrm{CCO}$ at $11 \mathrm{~K}$ [8.3211(1) $\AA$ A $[22,42,43]$. The change in lattice constant strongly influences the spin-phonon coupling in antiferromagnetic $\mathrm{Cr}$ spinel and modify the magnetic properties. The increase of lattice constant weakens the direct $\mathrm{Cr}-\mathrm{Cr}$ exchange interaction. The $\mathrm{Cr}-\mathrm{S}-\mathrm{Cr}$ and $\mathrm{Cr}-\mathrm{S}-\mathrm{Co}-\mathrm{S}-\mathrm{Cr}$ superexchange interactions become important [50]. According to the Goodenough-Kanamori rule for instance, a $90^{\circ}$ superexchange interaction between magnetic ion-ligand-magnetic ions usually provides ferromagnetic superexchange interactions for insulating magnetic materials [51]. Here, the $\mathrm{Cr}-\mathrm{S}-\mathrm{Cr}$ bond angle $\left(\sim 94^{\circ}\right)$ is close to $90^{\circ}$ as evident in Fig. 5(f) and indicates ferromagnetic superexchange according to the reported results [52]. This enhances the ferromagnetic component and, as a result of it, the bulk magnetic moment increases for CCS than CCO in accordance with the other members of $\mathrm{Cr}$ spinels [22,50]. Thus the structural aspect has the significant role for the magnetoelectric coupling, as magnetic and polar order are intimately correlated to the strong isostructural distortions.

\section{CONCLUSION}

In conclusion, the isostructural distortions of the spinel structure at $T_{N}$ and $T_{C}$ are the key for multiferroic order. For $\mathrm{CoCr}_{2} \mathrm{~S}_{4}$, the ferroelectric polarization provides the second largest value in $\mathrm{Cr}$-based spinels. Significant magnetoelectric coupling is observed involving occurrence of ferroelectric order and the magnetic field dependent dielectric permittivity scales linearly to the squared magnetization in the low field regime below $\sim 10 \mathrm{kOe}$ as described by the Ginzburg-Landau theory. The delicate interplay between lattice, spin, and charge degrees of freedom projects spinel sulphide $\mathrm{CoCr}_{2} \mathrm{~S}_{4}$ as an interesting system in improper multiferroics.

\section{ACKNOWLEDGMENTS}

S.G. acknowledges the financial support from DST, India (Project No. SB/S2/CMP-029/2014) and for the experiment at DESY, Germany under DST-DESY project for the synchrotron diffraction studies. S.G. also acknowledges Professor P. Mandal for providing the facility for the measurement of magnetodielectric response and Nanoscience unit of IACS, Kolkata for the MPMS facilities. K.D. wishes to thank CSIR, India for the fellowship.
[1] N. A. Spaldin, S.-W. Cheong, and R. Ramesh, Phys. Today 63, 38 (2010).

[2] M. Fiebig, J. Phys. D: Appl. Phys. 38, 123 (2005).

[3] S.-W. Cheong and M. Mostovoy, Nature Mater. 6, 13 (2007).

[4] J. F. Scott, J. Mater. Chem. 22, 4567 (2012).

[5] K. Dey, S. Majumdar, and S. Giri, Phys. Rev. B 90, 184424 (2014)

[6] K. Singh, A. Maignan, C. Simon, and C. Martin, Appl. Phys. Lett. 99, 172903 (2011).

[7] Y. Yamasaki, S. Miyasaka, Y. Kaneko, J.-P. He, T. Arima, and Y. Tokura, Phys. Rev. Lett. 96, 207204 (2006).

[8] Y. J. Choi, J. Okamoto, D. J. Huang, K. S. Chao, H. J. Lin, C. T. Chen, M. van Veenendaal, T. A. Kaplan, and S.-W. Cheong, Phys. Rev. Lett. 102, 067601 (2009).

[9] S. Yang, H. X. Bao, D. Z. Xue, C. Zhou, J. H. Gao, Y. Wang, J. Q. Wang, X. P. Song, Z. B. Sun, X. B. Ren, and K. Otsuka, J. Phys. D: Appl. Phys. 45, 265001 (2012).

[10] A. Maignan, C. Martin, K. Singh, Ch. Simon, O. I. Lebedev, and S. Turner, J. Solid State Chem. 195, 41 (2012).
[11] A. A. Bush, V. Y. Shkuratov, K. E. Kamentsev, A. S. Prokhorov, E. S. Zhukova, B. P. Gorshunov, and V. I. Torgashev, Phys. Rev. B 85, 214112 (2012).

[12] J. Hemberger, P. Lunkenheimer, R. Fichtl, H.-A. Krug von Nidda, V. Tsurkan, and A. Loidl, Nature (London) 434, 364 (2005).

[13] G. Catalan and J. F. Scott, Nature (London) 448, E4 (2007).

[14] S. Weber, P. Lunkenheimer, R. Fichtl, J. Hemberger, V. Tsurkan, and A. Loidl, Phys. Rev. Lett. 96, 157202 (2006).

[15] L. Lin, H. X. Zhu, X. M. Jiang, K. F. Wang, S. Dong, Z. B. Yan, Z. R. Yang, J. G. Wan, and J.-M. Liu, Sci. Rep. 4, 6530 (2015).

[16] K. Singh, A. Maignan, C. Martin, and Ch. Simon, Chem. Mater. 21, 5007 (2009).

[17] R. S. Preethi Meher, C. Martin, V. Caignaert, F. Damay, and A. Maignan, Chem. Mater. 26, 830 (2014).

[18] A. Karmakar, K. Dey, S. Chatterjee, S. Majumdar, and S. Giri, Appl. Phys. Lett. 104, 052906 (2014).

[19] L. K. Shick and A. R. Von Neida, J. Cryst. Growth 5, 313 (1969). 
[20] V. Sagredo, M. C. Morón, and G. E. Delgado, Physica B 384, 82 (2006).

[21] P. Gibart, M. Robbins, and V. G. Lambrecht Jr., J. Phys. Chem. Solids 34, 1363 (1973).

[22] K. Ohgushi, Y. Okimoto, T. Ogasawara, S. Miyasaka, and Y. Tokura, J. Phys. Soc. Jpn. 77, 034713 (2008).

[23] E. Carnall Jr., D. Pearlman, T. J. Coburn, F. Moser, and T. W. Martin, Mater. Res. Bull. 7, 1361 (1972).

[24] R. K. Ahrenkiel, T. H. Lee, S. L. Lyu, and F. Moser, Solid State Commun. 12, 1113 (1973).

[25] R. K. Aahrenkiel, T. J. Conurn, and E. Carnall Jr., IEEE Trans. Magn. 10, 2 (1974).

[26] R. K. Ahrenkiel, S. L. Lyu, and T. J. Coburn, J. Appl. Phys. 46, 894 (1975).

[27] T. Ogasawara, K. Ohgushi, Y. Tomioka, K. S. Takahashi, H. Okamoto, M. Kawasaki, and Y. Tokura, Phys. Rev. Lett. 94, 087202 (2005).

[28] T. Ogasawara, K. Ohgushi, H. Okamoto, and Y. Tokura, J. Phys. Soc. Jpn. 75, 083707 (2006).

[29] I. Kim, Y. S. Oh, Y. Liu, S. H. Chun, J.-S. Lee, K.-T. Ko, J.-H. Park, J.-H. Chung, and K. H. Kim, Appl. Phys. Lett. 94, 042505 (2009).

[30] T. Watanabe, Solid State Commun. 12, 355 (1973).

[31] T. Kimura, S. Kawamoto, I. Yamada, M. Azuma, M. Takano, and Y. Tokura, Phys. Rev. B 67, 180401(R) (2003).

[32] T. D. Sparks, M. C. Kemei, P. T. Barton, R. Seshadri, E.-D. Mun, and V. S. Zapf, Phys. Rev. B 89, 024405 (2014).

[33] N. Mufti, A. A. Nugroho, G. R. Blake, and T. T. M. Palstra, J. Phys. Condens. Matter 22, 075902 (2010).

[34] R. Saha, A. Sundaresan, and C. N. R. Rao, Mater. Horiz. 1, 20 (2014).

[35] A. Ghosh, A. Pal, K. Dey, S. Majumdar, and S. Giri, J. Mater. Chem. C 3, 4162 (2015).

[36] K. R. S. Preethi Meher, A. Wahl, A. Maignan, C. Martin, and O. I. Lebedev, Phys. Rev. B 89, 144401 (2014).
[37] S. Chowki, T. Basu, K. Singh, N. Mohapatra, and E. V. Sampathkumaran, J. Appl. Phys. 115, 214107 (2014).

[38] K. Singh, T. Basu, S. Chowki, N. Mahapotra, K. K. Iyer, P. L. Paulose, and E. V. Sampathkumaran, Phys. Rev. B 88, 094438 (2013).

[39] J. Sannigrahi, S. Chattopadhyay, D. Dutta, S. Giri, and S. Majumdar, J. Phys. Condens. Mater 25, 246001 (2013).

[40] S. Lafuerza, J. García, G. Subías, J. Blasco, K. Conder, and E. Pomjakushina, Phys. Rev. B 88, 085130 (2013).

[41] N. Ikeda, H. Ohsumi, K. Ohwada, K. Ishii, T. Inami, K. Kakurai, Y. Murakami, K. Yoshii, S. Mori, Y. Horibe, and H. Kito, Nature (London) 436, 1136 (2005).

[42] P. K. Baltzer, P. J. Wojtowxcz, M. Robbins, and E. Lqpatin, Phys. Rev. 151, 367 (1966).

[43] G. Lawes, B. Melot, K. Page, C. Ederer, M. A. Hayward, Th. Proffen, and R. Seshadri, Phys. Rev. B 74, 024413 (2006).

[44] L. J. Chang, D. J. Huang, W.-H. Li, S.-W. Cheong, W. Ratcliff, and J. W. Lynn, J. Phys. Condens. Matter 21, 456008 (2009).

[45] K. Dey, A. Karmakar, S. Majumdar, and S. Giri, Phys. Rev. B 87, 094403 (2013).

[46] K. Dey, A. Karmakar, S. Majumdar, and S. Giri, Scr. Mater. 75, 94 (2014).

[47] A. Marais, M. Porte, L. Goldstein, and P. Gibart, J. Magn. Magn. Mater. 15-18, 1287 (1980).

[48] M. R. Suchomel, D. P. Shoemaker, L. Ribaud, M. C. Kemei, and R. Seshadri, Phys. Rev. B 86, 054406 (2012).

[49] K. Tomiyasu, J. Fukunaga, and H. Suzuki, Phys. Rev. B 70, 214434 (2004).

[50] T. Rudolf, Ch. Kant, F. Mayr, J. Hemberger, V. Tsurkan, and A. Loidl, New J. Phys. 9, 76 (2007).

[51] J. Goodenough, Magnetism and the Chemical Bond (Wiley, New York, 1963).

[52] P. Gibart, J.-L. Dormann, and Y. Pellerin, Phys. Status Solidi 36, 187 (1969). 